\title{
Blind Image Deblurring Driven by Nonlinear Processing in the Edge Domain
}

\author{
Stefania Colonnese \\ Dipartimento Infocom, Università degli Studi di Roma "La Sapienza," Via Eudossiana 18, 00184 Roma, Italy
}

Email: colonnese@infocom.uniroma1.it

Patrizio Campisi

Dipartimento Elettronica Applicata, Università degli Studi “Roma Tre," Via Della Vasca Navale 84, 00146 Roma, Italy Email:campisi@uniroma3.it

\author{
Gianpiero Panci \\ Dipartimento Infocom, Università degli Studi di Roma "La Sapienza," Via Eudossiana 18, 00184 Roma, Italy \\ Email:gpanci@infocom.uniroma1.it
}

\author{
Gaetano Scarano \\ Dipartimento Infocom, Università degli Studi di Roma "La Sapienza," Via Eudossiana 18, 00184 Roma, Italy \\ Email: scarano@infocom.uniroma1.it
}

Received 2 September 2003; Revised 20 February 2004

\begin{abstract}
This work addresses the problem of blind image deblurring, that is, of recovering an original image observed through one or more unknown linear channels and corrupted by additive noise. We resort to an iterative algorithm, belonging to the class of Bussgang algorithms, based on alternating a linear and a nonlinear image estimation stage. In detail, we investigate the design of a novel nonlinear processing acting on the Radon transform of the image edges. This choice is motivated by the fact that the Radon transform of the image edges well describes the structural image features and the effect of blur, thus simplifying the nonlinearity design. The effect of the nonlinear processing is to thin the blurred image edges and to drive the overall blind restoration algorithm to a sharp, focused image. The performance of the algorithm is assessed by experimental results pertaining to restoration of blurred natural images.
\end{abstract}

Keywords and phrases: blind image restoration, Bussgang deconvolution, nonlinear processing, Radon transform.

\section{INTRODUCTION}

Image deblurring has been widely studied in literature because of its theoretical as well as practical importance in fields such as astronomical imaging [1], remote sensing [2], medical imaging [3], to cite only a few. Its goal consists in recovering the original image from a single or multiple blurred observations.

In some application cases, the blur is assumed known, and well-known deconvolution methods, such as Wiener filtering, recursive Kalman filtering, and constrained iterative deconvolution methods, are fruitfully employed for restoration.

However, in many practical situations, the blur is partially known [4] or unknown, because an exact knowledge of the mechanism of the image degradation process is not avail- able. Therefore, the blurring action needs to be characterized on the basis of the available blurred data, and blind image restoration techniques have to be devised for restoration. These techniques aim at the retrieval of the image of interest observed through a nonideal channel whose characteristics are unknown or partially known in the restoration phase. Many blind restoration algorithms have been proposed in the past, and an extended survey can be found in $[5,6]$.

In some applications, the observation system is able to give multiple observations of the original image. In electron microscopy, for example, many differently focused versions of the same image are acquired during a single experiment, due to an intrinsic tradeoff between the bandwidth of the imaging system and the contrast of the resulting image. In other applications, such as telesurveillance, multiple observed images can be acquired in order to better counteract, 


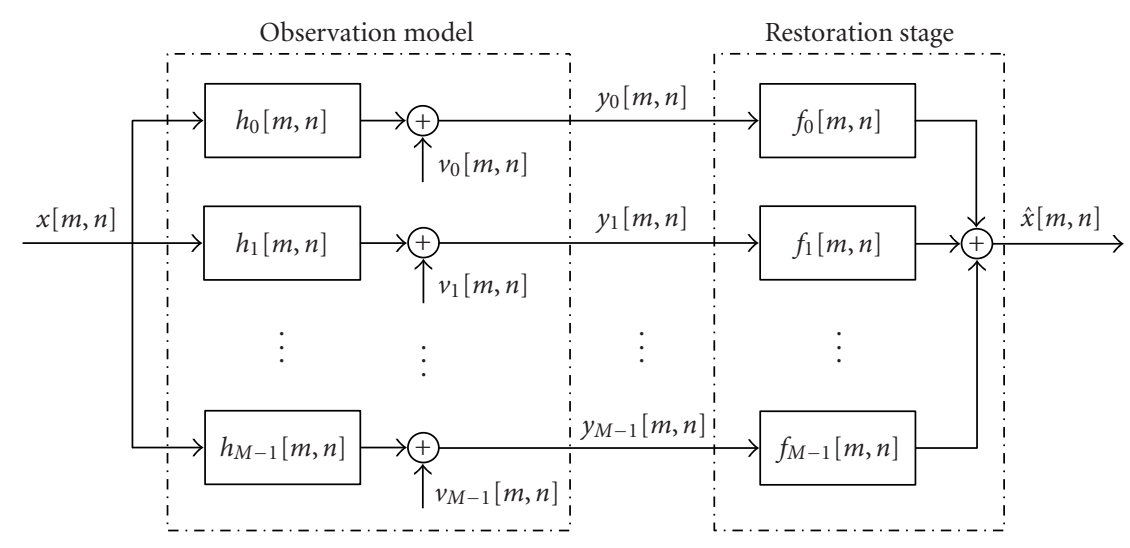

Figure 1: Blurred image generation model and restoration stage.

in the restoration phase, possible degradation due to motion, defocus, or noise. In remote sensing applications, by employing sensor diversity, different versions of the same scene can be acquired at different times through the atmosphere that can be modeled as a time-variant channel.

Different approaches have been proposed in the recent past to face the image deblurring problem. In [7], it is shown that, under some mild assumptions, both the filters and the image can be exactly determined from noise-free observations as well as stably estimated from noisy observations. Both in $[7,8]$, the channel estimation phase precedes the restoration phase. Once the channel has been estimated, image restoration is performed by subspace-based and likelihood-based algorithms [7], or by a bank of finite impulse response (FIR) filters optimized with respect to a deterministic criterion [8].

Different approaches resort to suitable image representation domains. To cite a few, in [9], a wavelet-based edge preserving regularization algorithm is presented, while in [10], the image restoration is accomplished using simulated annealing on a suitably restricted wavelet space. In [11], the authors make use of the Fourier phase for image restoration [12] applying appropriate constraints in the Radon domain.

In $[13,14]$, the authors resort to an iterative algorithm, belonging to the class of Bussgang algorithms, based on alternating a linear and a nonlinear image estimation stage. The nonlinear estimation phase plays a key role in the overall algorithm since it attracts the estimate towards a final restored image possessing some desired structural or statistical characteristics. The design of the nonlinear processing stage is aimed at superimposing the desired characteristics on the restored image. While for class of images with known probabilistic description, such as text images, the nonlinearity design can be conducted on the basis of a Bayesian criterion, for natural images, the image characterization and hence the nonlinearity design is much more difficult. In [14], the authors design the nonlinear processing in a transformed domain that allows a compact representation of the image edges - the edge domain.
In this paper, we investigate the design of the nonlinear processing stage using the Radon Transform (RT) [15] of the image edges. This choice is motivated by the fact that the RT of the image edges well describes the structural image features and the effect of blur, thus simplifying the nonlinearity design.

The herein discussed approach shares some common points with [16] since it exploits a compact multiscale representation of natural images.

The structure of the paper is as follows. In Section 2, the observation model is described. Following the recent literature, a multichannel approach is pursued. Section 3 recalls the basic outline of the Bussgang algorithm, which is described in detail in the appendix. Section 4 is devoted to the description of the image edge extraction as well as to the discussion of the nonlinearity design in the edge domain. Section 5 presents the results of the blind restoration algorithm and Section 6 concludes the paper.

\section{THE OBSERVATION MODEL}

The single-input multiple-output (SIMO) observation model of images is represented by $M$ linear observation filters in presence of additive noise. This model, depicted in Figure 1, is given by

$$
\begin{aligned}
y_{0}[m, n] & =\left(x * h_{0}\right)[m, n]+v_{0}[m, n], \\
y_{1}[m, n] & =\left(x * h_{1}\right)[m, n]+v_{1}[m, n], \\
\vdots & \\
y_{M-1}[m, n] & =\left(x * h_{M-1}\right)[m, n]+v_{M-1}[m, n],
\end{aligned}
$$

where $x$ denotes the whole image, $x[n, m]$ represents either the whole image or $n$ th, $m$ th pixels of the image $x$, depending on the context, and $x * h$ refers to the whole image resulting after convolution. Moreover, let $v_{i}[m, n], i=0, \ldots, M-$ 1 , be realizations of mutually uncorrelated, white Gaussian 


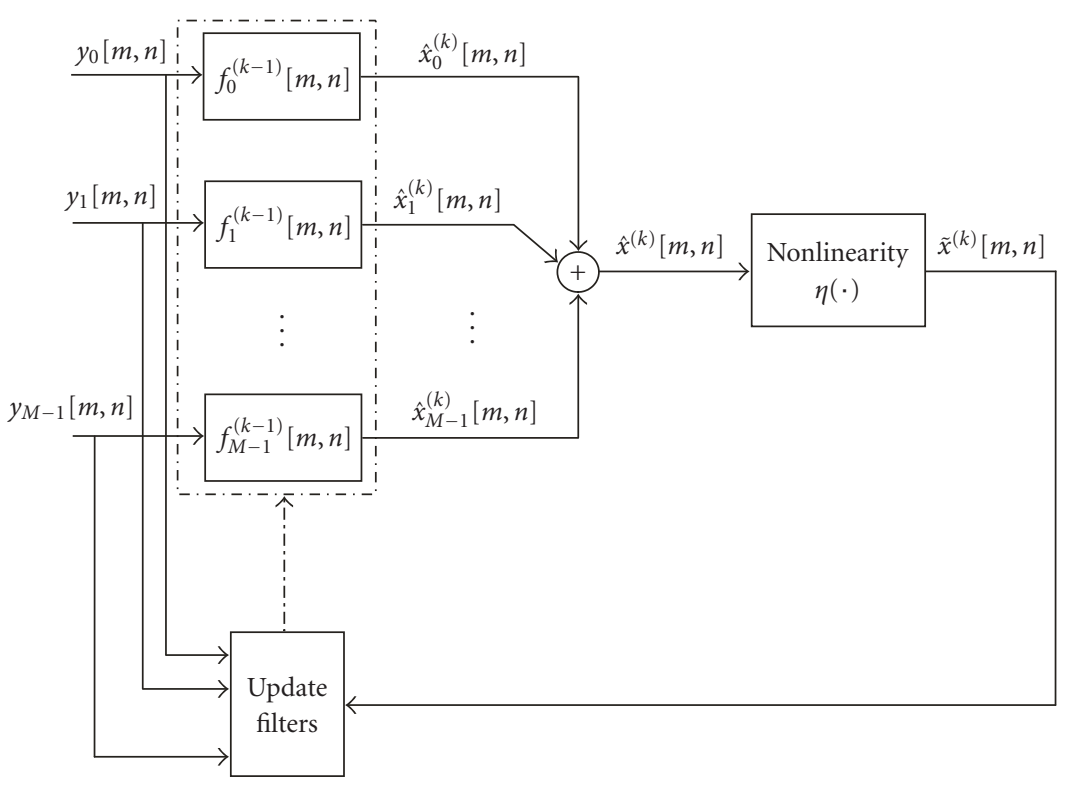

FIGURE 2: General form of the Bussgang deconvolution algorithm.

processes, that is,

$$
\begin{aligned}
\mathrm{E}\left\{v_{i}[m, n] v_{j}[m-r, n-s]\right\} & =\sigma_{v_{i}}^{2} \delta[r, s] \cdot \delta[i-j] \\
& = \begin{cases}\sigma_{v_{i}}^{2} \cdot \delta[r, s] & \text { for } i=j, \\
0 & \text { for } i \neq j .\end{cases}
\end{aligned}
$$

Here, $\mathrm{E}\{\cdot\}$ represents the expected value, $\delta[\cdot]$ the unit sample, and $\delta[\cdot, \cdot]$ the bidimensional unit sample.

\section{MULTICHANNEL BUSSGANG ALGORITHM}

The basic structure of one step of the iterative Bussgang algorithm for blind channel equalization [17, 18, 19], or blind image restoration $[14,20]$, consists of a linear filtering of the measurements, followed by a nonlinear processing of the filter output, and concluded by updating the filter coefficients using both the measurements and the output of the nonlinear processor. The scheme of the iterative multichannel Bussgang blind deconvolution algorithm, as presented in [14], is depicted in Figure 2. The linear restoration stage is accomplished using a bank of FIR restoration filters $f_{i}^{(k)}[m, n]$, $i=0, \ldots, M-1$, with finite support of size $(2 P+1) \times(2 P+1)$, namely,

$$
\begin{aligned}
\hat{x}^{(k)}[m, n] & =\sum_{i=0}^{M-1}\left(y_{i} * f_{i}^{(k)}\right)[m, n] \\
& =\sum_{i=0}^{M-1} \sum_{t, u=-P}^{P} f_{i}^{(k)}[t, u] y_{i}[m-t, n-u] .
\end{aligned}
$$

At each iteration, a nonlinear estimate $\tilde{x}^{(k)}[m, n]=$ $\eta\left(\hat{x}^{(k)}[m, n]\right)$ is then obtained from $\hat{x}^{(k)}[m, n]$. Then, the filter coefficients are updated by solving a linear system (normal equations) whose coefficients' matrix takes into account the cross-correlation between the observations $y_{i}[m, n], i=$ $0, \ldots, M-1$, and the nonlinear estimate of the original image $\tilde{x}^{(k)}[m, n]$. A description of the algorithm is reported in the appendix.

\section{BUSSGANG NONLINEARITY DESIGN IN THE EDGE DOMAIN USING THE RADON TRANSFORM}

The quality of the restored image obtained by means of the Bussgang algorithm strictly depends on how the adopted nonlinear processing is able to restore specific characteristics or properties of the original image. If the unknown image is well characterized using a probabilistic description, as for text images, the nonlinearity $\eta(\cdot)$ can be designed on the basis of a Bayesian criterion, as the "best" estimate of $x[m, n]$ given $\hat{x}^{(k)}[m, n]$. Often, the minimum mean square error (MMSE) criterion is adopted.

For natural images, we design the nonlinearity $\eta(\cdot)$ after having represented the linear estimate ${ }^{1} \hat{x}[m, n]$ in a transformed domain in which both the blur effect and the original image structural characteristics are easily understood.

We consider the decomposition of the linear estimate $\hat{x}[m, n]$ by means of a filter pair composed of the lowpass filter $\psi^{(0)}[m, n]$ and a bandpass filter $\psi^{(1)}[m, n]$ (see Figure 3 ) whose impulse responses are

$$
\begin{aligned}
\psi^{(0)}[m, n] & =e^{-r^{2}[m, n] / \sigma_{0}^{2}}, \\
\psi^{(1)}[m, n] & =\frac{r[m, n]}{\sigma_{1}} e^{-r^{2}[m, n] / \sigma_{1}^{2}} e^{-j \theta[m, n]},
\end{aligned}
$$

where $r[m, n] \stackrel{\text { def }}{=} \sqrt{m^{2}+n^{2}}$ and $\theta[m, n] \stackrel{\text { def }}{=} \arctan n / m$ are

\footnotetext{
${ }^{1}$ To simplify the notation, in the following, we will drop the superscript (k) referring to the $k$ th iteration of the deconvolution algorithm.
} 


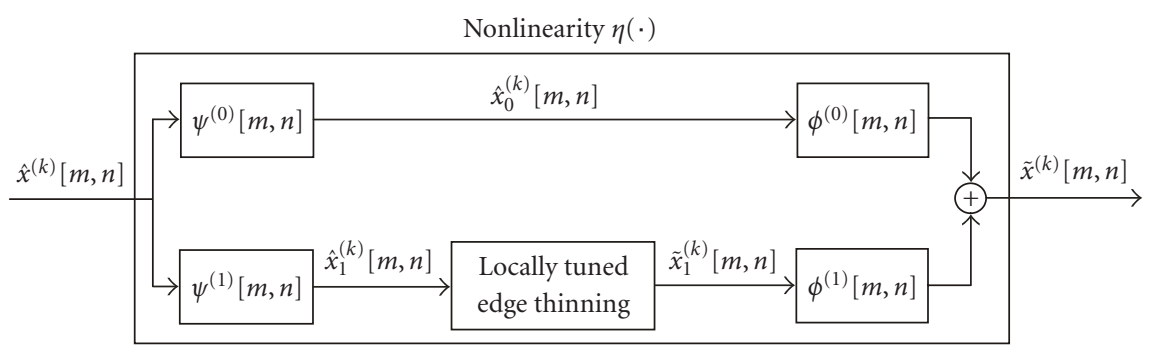

FIGURE 3: Multichannel nonlinear estimator $\eta(\cdot)$.

discrete polar pixel coordinates. These filters belong to the class of the circular harmonic functions (CHFs), whose detailed analysis can be found in $[21,22]$, and possess the interesting characteristic of being invertible by a suitable filter pair $\phi^{(0)}[m, n], \phi^{(1)}[m, n]$.

For the values of the form factors $\sigma_{0}$ and $\sigma_{1}$ of interest, the corresponding transfer functions can be well approximated as follows:

$$
\begin{aligned}
& \Psi^{(0)}\left(e^{j \omega_{1}}, e^{j \omega_{2}}\right) \simeq \pi \sigma_{0}^{2} e^{-\rho^{2}\left(\omega_{1}, \omega_{2}\right) \sigma_{0}^{2} / 4}, \\
& \Psi^{(1)}\left(e^{j \omega_{1}}, e^{j \omega_{2}}\right) \simeq \frac{-j \pi \sigma_{1}^{3}}{2} \rho\left(\omega_{1}, \omega_{2}\right) e^{-\rho^{2}\left(\omega_{1}, \omega_{2}\right) \sigma_{1}^{2} / 4} e^{-j \gamma\left(\omega_{1}, \omega_{2}\right)},
\end{aligned}
$$

$\rho\left(\omega_{1}, \omega_{2}\right) \stackrel{\text { def }}{=} \sqrt{\omega_{1}^{2}+\omega_{2}^{2}}$, and $\gamma\left(\omega_{1}, \omega_{2}\right) \stackrel{\text { def }}{=} \arctan \omega_{2} / \omega_{1}$, being the polar coordinates in the spatial radian frequency domain.

The reconstruction filters $\phi^{(0)}[m, n]$ and $\phi^{(1)}[m, n]$ satisfy the invertibility condition $\Psi^{(0)}\left(e^{j \omega_{1}}, e^{j \omega_{2}}\right) \cdot \Phi^{(0)}\left(e^{j \omega_{1}}, e^{j \omega_{2}}\right)+$ $\Psi^{(1)}\left(e^{j \omega_{1}}, e^{j \omega_{2}}\right) \cdot \Phi^{(1)}\left(e^{j \omega_{1}}, e^{j \omega_{2}}\right)=1$.

By indicating with $\overline{(\cdot)}$ the complex conjugate operator, in the experiments, we have chosen

$$
\begin{aligned}
& \Phi^{(0)}\left(e^{j \omega_{1}}, e^{j \omega_{2}}\right)=\frac{\overline{\Psi^{(0)}}\left(e^{j \omega_{1}}, e^{j \omega_{2}}\right)}{\left|\Psi^{(0)}\left(e^{j \omega_{1}}, e^{j \omega_{2}}\right)\right|^{2}+\left|\Psi^{(1)}\left(e^{j \omega_{1}}, e^{j \omega_{2}}\right)\right|^{2}}, \\
& \Phi^{(1)}\left(e^{j \omega_{1}}, e^{j \omega_{2}}\right)=\frac{\overline{\Psi^{(1)}}\left(e^{j \omega_{1}}, e^{j \omega_{2}}\right)}{\left|\Psi^{(0)}\left(e^{j \omega_{1}}, e^{j \omega_{2}}\right)\right|^{2}+\left|\Psi^{(1)}\left(e^{j \omega_{1}}, e^{j \omega_{2}}\right)\right|^{2}}
\end{aligned}
$$

to prevent amplification of spurious components occurring at those spatial frequencies, where $\Psi^{(0)}\left(e^{j \omega_{1}}, e^{j \omega_{2}}\right)$ and $\Psi^{(1)}\left(e^{j \omega_{1}}, e^{j \omega_{2}}\right)$ are small in magnitude. The optimality of these reconstruction filters is discussed in [23].

The zero-order circular harmonic filter $\psi^{(0)}[m, n]$ extracts a lowpass version $\hat{x}_{0}[m, n]$ of the input image; the form factor $\sigma_{0}$ is chosen so to retain only very low spatial frequencies, so obtaining a lowpass component exhibiting high spatial correlation. The first-order circular harmonic filter $\psi^{(1)}[m, n]$ is a bandpass filter, with frequency selectivity set by properly choosing the form factor $\sigma_{1}$. The output of this filter is a complex image $\hat{x}_{1}[m, n]$, which will be referred to in the following as "edge image," whose magnitude is related to the presence of edges and whose phase is proportional to their orientation.
Coarsely speaking, the edge image $\hat{x}_{1}[m, n]$ is composed of curves, representing edges occurring in $x[m, n]$, whose width is controlled by the form factor $\sigma_{1}$, and of low magnitude values representing the interior of uniform or textured regions occurring in $x[m, n]$. Strong intensity curves in $\hat{x}_{1}[m, n]$ are well analyzed by the local application of the bidimensional RT. This transform maps a straight line into a point in the transformed domain, and therefore it yields a compact and meaningful representation of the image's edges. However, since most image's edges are curves, the analysis must be performed locally by partitioning the image into regions small enough such that in each block, only straight lines may occur. Specifically, after having chosen the region dimensions, the value of the filter parameter $\sigma_{1}$ is set such that the width of the observed curve is a small fraction of its length. In more detail, the evaluation of the edge image is performed by the $\mathrm{CH}$ filter of order one $\psi^{(1)}[m, n]$ that can be seen as the cascade of a derivative filter followed by a Gaussian smoothing filter. The response to an abrupt edge of the original image is a line in $\hat{x}_{1}[m, n]$. The line is centered in correspondence to the edge, whose energy is concentrated in an interval of $\pm \sigma_{1}^{-1}$ pixels and that slowly decays to zero in an interval of $\pm 3 \sigma_{1}^{-1}$ pixels. Therefore, by partitioning the image into blocks of $8 \times 8$ pixels, the choice of $\sigma_{1} \approx 1$ yields edge structures that are well readable in the partitions of the edge image.

Then each region is classified as either a "strong edge" region or as a "weak edge" and "textured" region. The proposed enhancement procedures for the different kinds of regions are described in detail in Section 4.2.

It is worth pointing out that our approach shares the local RT as common tool with a family of recently proposed image transforms - the curvelet transforms [16, 24, 25]that represent a significant alternative to wavelet representation of natural images. In fact, the curvelet transform yields a sparse representation of both smooth image and edges, either straight or curved.

\subsection{Local Radon transform of the edge image: original image and blur characterization}

The edge image is a sparse complex image built by a background of zero or low magnitude areas, in which the objects appearing in the original image domain are sketched by their edges. 
We discuss here this representation in more detail. For a continuous image $\xi\left(t_{1}, t_{2}\right)$, the RT [15] is defined as

$$
\begin{array}{r}
p_{\beta}^{\xi}(s) \stackrel{\text { def }}{=} \int_{-\infty}^{\infty} \xi(\cos \beta \cdot s-\sin \beta \cdot u, \sin \beta \cdot s+\cos \beta \cdot u) d u \\
-\infty<s<\infty, \beta \in[0, \pi)
\end{array}
$$

that represents the summation of $\xi\left(t_{1}, t_{2}\right)$ along a ray at distance $s$ and angle $\beta$.

It is well known [15] that it can be inverted by

$$
\xi\left(t_{1}, t_{2}\right)=\frac{1}{4 \pi^{2}} \int_{0}^{\pi} \int_{-\infty}^{\infty} P_{\beta}^{\xi}(j \sigma) e^{j \sigma\left(\sigma \cos \beta t_{1}+\sigma \sin \beta t_{2}\right)}|\sigma| d \sigma d \beta
$$

where $P_{\beta}^{\xi}(j \sigma)=\mathcal{F}\left\{p_{\beta}^{\xi}(s)\right\}$ is the Fourier transform of the RT. Note that $\mathcal{F}\{\cdot\}$ represents the Fourier transform.

Some details about the discrete implementation of the RT follows.

If the image $\xi\left(t_{1}, t_{2}\right)$ is frequency limited in a circle of diameter $D_{\Omega}$, it can be reconstructed by the samples of its RT taken at spatial sampling interval $\Delta s \leq 2 \pi / D_{\Omega}$,

$$
p_{\beta}^{\xi}[n]=\left.p_{\beta}^{\xi}(s)\right|_{s=n \cdot \Delta s}, \quad n=0, \pm 1, \pm 2, \ldots
$$

Moreover, if the original image is approximately limited in the spatial domain, that is, it vanishes out of a circle of diameter $D_{t}$, the sequence $p_{\beta}^{\xi}[n]$ has finite length $N=1+D_{t} / \Delta s$. In a similar way, the RT can be sampled with respect to the angular parameter $\beta$ considering $M$ different angles $m \Delta \beta$, $m=0, \ldots, M-1$, with sampling interval $\Delta \beta$, namely,

$$
p_{\beta_{m}}^{\xi}[n]=\left.p_{\beta_{m}}^{\xi}(s)\right|_{s=n \cdot \Delta s, \beta_{m}=m \cdot \Delta \beta}
$$

The angular interval $\Delta \beta$ can be chosen so as to assure that the distance between points $p_{\beta}^{\xi}[n]$ and $p_{\beta+\Delta \beta}^{\xi}[n]$ lying on adjacent diameters remains less than or equal to the chosen spatial sampling interval $\Delta s$, that is,

$$
\Delta \beta \cdot \frac{D_{t}}{2} \leq \Delta s
$$

The above condition is satisfied when $M \geq(\pi / 2) \cdot N \simeq 1.57$. $N$.

As long as the edge image is concerned, each region is here modeled as obtained by ideal sampling of an original image $x_{1}\left(t_{1}, t_{2}\right)$, approximately spatially bounded by a circle of diameter $D_{t}$, and bandwidth limited in a circle of diameter $D_{\Omega}$. Under the hypothesis that $N-1 \geq D_{t} \cdot D_{\Omega} / 2 \pi$, and $M \geq$ $(\pi / 2) \cdot N$, the $M, N$ samples

$$
p_{\beta_{m}}^{x_{1}}[n], \quad m=0, \ldots, M-1, n=0, \ldots, N-1,
$$

of the RT $p_{\beta}^{x_{1}}(s)$ allow the reconstruction of the image $x_{1}\left(t_{1}, t_{2}\right)$, and hence of any pixel of the selected region.

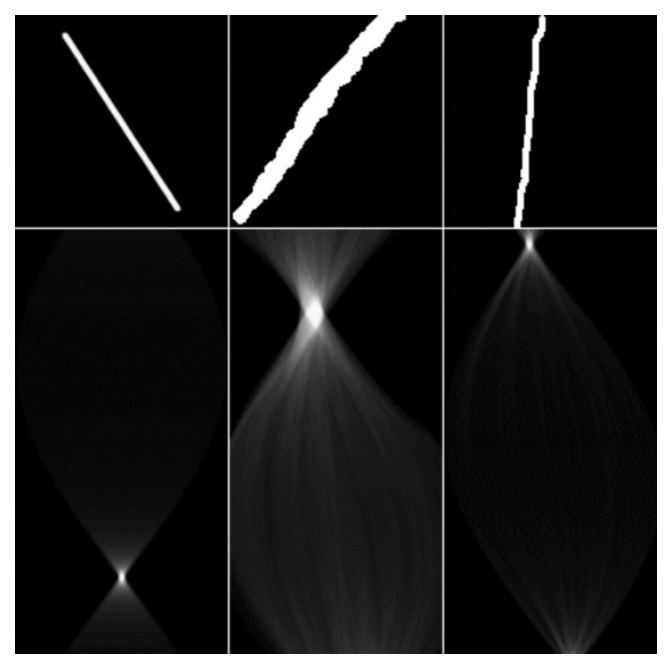

FIGURE 4: First row: original edges. Second row: corresponding discrete Radon transform.

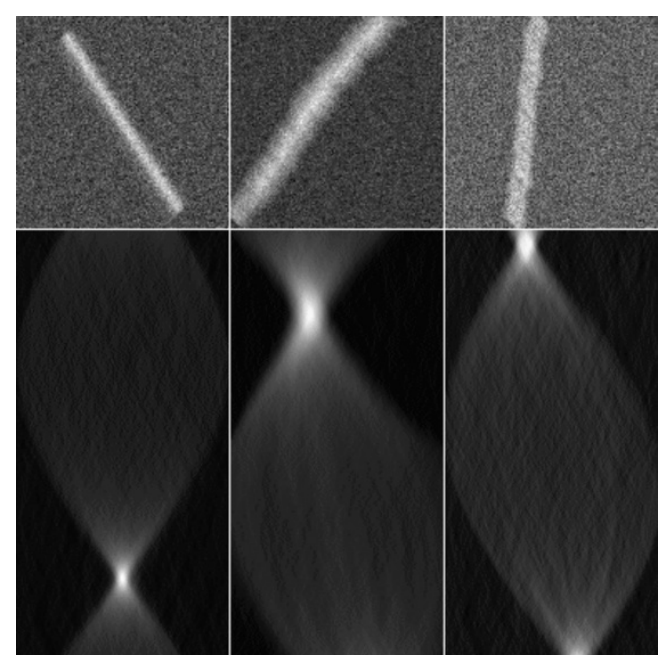

FIGURE 5: First row: blurred edges. Second row: corresponding discrete Radon transforms.

In Figure 4, some examples of straight edges and their corresponding discrete RT are shown.

We now consider the case of blurred observations. In the edge image, the blur tends to flatten and attenuate the edge peaks, and to smooth the edge contours in directions depending on the blur itself. The effects of blur on the RT of the edge image regions are primarily two. The first effect is that, since the energy of each edge is spread in the spatial domain, the maximum value of the RT is lowered. The second effect is that, since the edge width is typically thickened, it contributes to different tomographic projections, enhancing two triangular regions in the RT. This behavior is illustrated by the example in Figure 5, where a motion blur filter is applied to an original edge.

Stemming from this compact representation of the blur effect, we will devise an effective nonlinearity aimed at restoring the original edge. 


\subsection{Local Radon transform of the edge image: nonlinearity design}

The design of the nonlinearity will be conducted after having characterized the blur effect at the output of a first-order CHF bank. By choosing the form factor $\sigma_{0}$ of the zero-order $\mathrm{CH}$ filter $\psi^{(0)}$ small enough, in the passband, the blur transfer function is approximately constant, and thus the blur effect on the lowpass component is negligible.

As far as the first-order $\mathrm{CH}$ filter's domain is concerned, the blur causes the edges in the spatial domain to be spread along directions depending on the impulse responses of the blurring filters. After having partitioned the edge image into small regions in order to perform a local RT as detailed in Section 4.1, each region has to be classified either as a strong edge area or a weak edge and textured area. Hence, the nonlinearity has to be adapted to the degree of "edgeness" of each region in which the image has been partitioned. The decision rule between the two areas is binary. Specifically, an area characterized as a "strong edge" region has an RT whose coefficients assume significant values only on a subset of directions $\beta_{m}$. Therefore, a region is classified as a "strong edge" area by comparing $\max _{m} \sum_{n}\left(p_{\beta_{m}}^{\xi}[n]\right)^{2}$ with a fixed threshold. If the threshold is exceeded, the area is classified as a strong edge area; otherwise, this implies that either no direction is significant, which corresponds to weak edges, or every direction is equally significant, which corresponds to textured areas.

\section{Strong edges}

For significant image edges, characterized by relevant energy concentrated in one direction, the nonlinearity can exploit the spatial memory related to the edge structure. In this case, as above discussed, we use the RT of the edge image. We consider a limited area of the edge image $\hat{x}_{1}[m, n]$ intersected by an edge, and its RT $p_{\beta_{m}}^{\hat{x}_{1}}[m, n]$, with $m, n$ chosen as discussed in Section 4.1.

The nonlinearity we present aims at focusing the RT both with respect to $m$ and $n$, and it is given by

$$
p_{\beta_{m}}^{\tilde{x}_{1}}[n]=p_{\beta_{m}}^{\hat{x}_{1}}[n] \cdot g^{\kappa_{g}}\left(\beta_{m}\right) \cdot f_{\beta_{m}}^{\kappa_{f}}(n)
$$

with

$$
\begin{aligned}
g\left(\beta_{m}\right) & =\frac{\max _{n}\left(p_{\beta_{m}}^{\hat{x}_{1}}[n]\right)-\min _{\beta_{k}, n}\left(p_{\beta_{k}}^{\hat{x}_{1}}[n]\right)}{\max _{\beta_{k}, n}\left(p_{\beta_{k}}^{\hat{x}_{1}}[n]\right)-\min _{\beta_{k}, n}\left(p_{\beta_{k}}^{\hat{x}_{1}}[n]\right)}, \\
f_{\beta_{m}}(n) & =\frac{p_{\beta_{m}}^{\hat{x}_{1}}[n]-\min _{n}\left(p_{\beta_{m}}^{\hat{x}_{1}}[n]\right)}{\max _{n}\left(p_{\beta_{m}}^{\hat{x}_{1}}[n]\right)-\min _{n}\left(p_{\beta_{m}}^{\hat{x}_{1}}[n]\right)},
\end{aligned}
$$

where $\max _{n}\left(p_{\beta_{m}}^{\hat{x}_{1}}[n]\right)$ and $\min _{n}\left(p_{\beta_{m}}^{\hat{x}_{1}}[n]\right)$ represent the maximum and the minimum value, respectively, of the RT for the direction $\beta_{m}$ under analysis, and $\max _{\beta_{k}, n}\left(p_{\beta_{k}}^{\hat{x}_{1}}[n]\right)$ and $\min _{\beta_{k}, n}\left(p_{\beta_{k}}^{\hat{x}_{1}}[n]\right)$, with $k=0, \ldots, M-1$, the global maximum and the global minimum, respectively, in the Radon domain. Therefore, for each point belonging to the direction $\beta_{m}$ and having index $n$, the nonlinearity (13) weights the

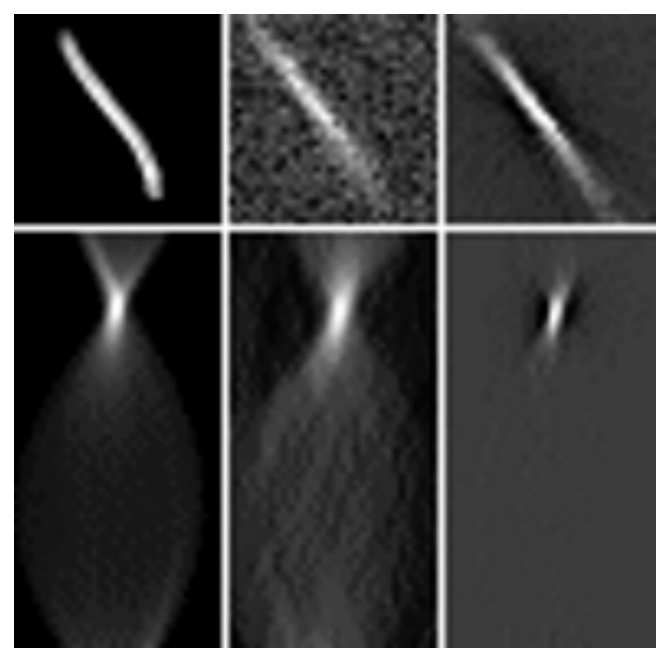

FIGURE 6: First row, from left to right: original edge, blurred edge, and restored edge. Second row: corresponding discrete Radon transforms.

RT by two gain functions. Specifically, (14) assumes its maximum value (equal to 1 ) for the direction $\beta_{\operatorname{Max}}$, where the global maximum occurs and it decreases for the other directions. In other words, (14) assigns a relative weight equal to 1 to the direction $\beta_{\text {Max }}$ whereas attenuates the other directions. Moreover, for a given direction $\beta_{m}$, (15) determines the relative weight of the actual displacement $n$ with respect to the others by assigning a weight equal to 1 to the displacement where the maximum occurs and by attenuating the other locations. The factors $\kappa_{g}$ and $\kappa_{f}$ in (14) and (15) are defined as $\kappa_{g}=\kappa_{0} \sigma_{w^{(k)}}^{2}$ and $\kappa_{f}=\kappa_{1} \sigma_{w^{(k)}}^{2}, \sigma_{w^{(k)}}^{2}$ being the deconvolution noise variance and $\kappa_{0}$ and $\kappa_{1}$ two constants empirically chosen and set for our experiments equal to 2.5 and 0.5 , respectively. The deconvolution noise variance $\sigma_{w^{(k)}}^{2}$ depends on both the blur and on the observation noise, and it can be estimated as $\mathrm{E}\left\{\left|w^{(k)}[m, n]\right|^{2}\right\} \approx \mathrm{E}\left\{\left|\tilde{x}^{(k)}[m, n]-\hat{x}[m, n]\right|^{2}\right\}$ when the algorithm begins to converge. The deconvolution noise variance gradually decreases at each iteration which guarantees a gradually decreasing action of the nonlinearity as the iterations proceed.

The edge enhancement in the Radon domain is then described by the combined action of (14) and (15), since the first estimates the edge direction and the second performs a thinning operation for that direction.

To depict the effect of the nonlinearity (13) on the edge domain, in Figure 6, the case of a straight edge is illustrated.

The first columns of Figures 7 and 8 show some details extracted from the edge images of blurred versions of the "F16" (Figure 9) and "Cameraman" (Figure 10) images, respectively. For each highlighted block of $8 \times 8$ pixels, the RT is calculated by considering the block as belonging to a circular region of diameter $8 \sqrt{2}$ (circumscribed circle). The above discussed nonlinearity is then applied. Then the inverse RT is evaluated for the pixels belonging to the central $8 \times 8$ pixels square. We observe that, although some pixels belong to two circles, namely, the circle related to the considered block 


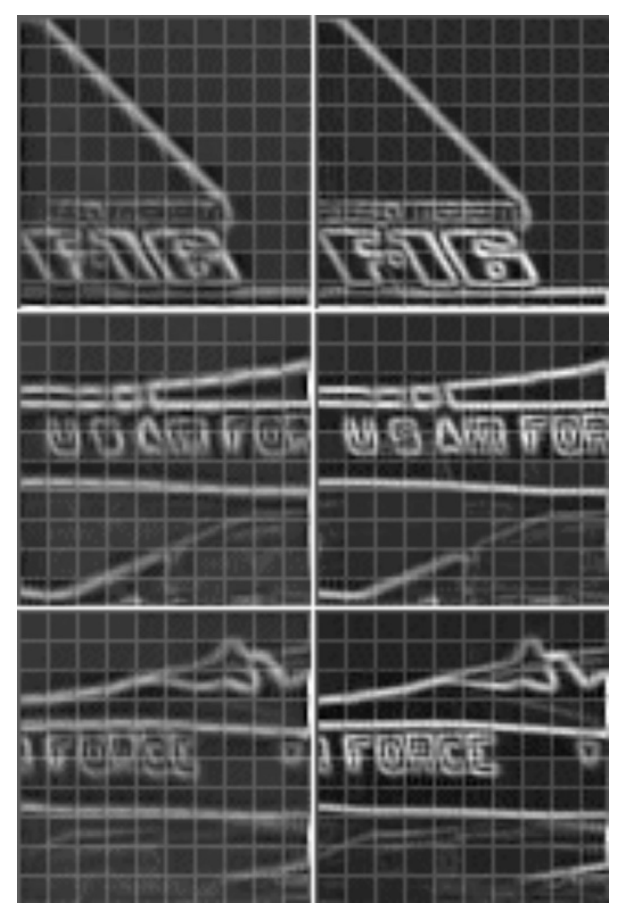

FIGURE 7: First column: details of the F16 blurred image in the edge domain. Second column: corresponding restored details in the edge domain.
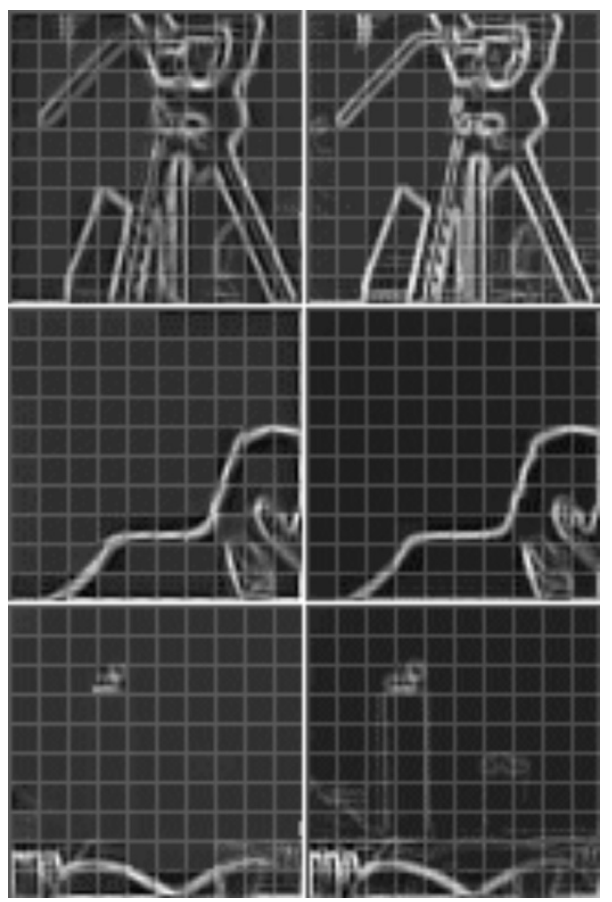

FIGURE 8: First column: details of the Cameraman blurred image in the edge domain. Second column: corresponding restored details in the edge domain.

and the circle related to the closest block, for each pixel, only the inverse RT relative to its own block is considered. The restored details in the edge domain are shown in the second

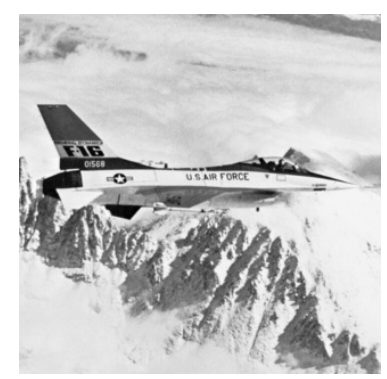

Figure 9: F16 image.

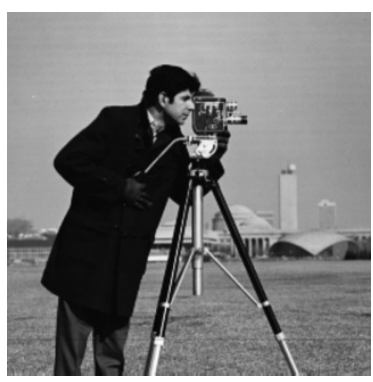

FIgure 10: Cameraman image.

columns. The edges are clearly enhanced and focused by the processing.

\section{Weak edges and textured regions}

If the image is flat or does not exhibit any directional structure able to drive the nonlinearity, we use a spatially zeromemory nonlinearity, acting pointwise on the edge image. Since the edge image is almost zero in every pixel corresponding to the interior of uniform regions, where small values are likely due to noise, the nonlinearity should attenuate low-magnitude values of $\hat{x}_{1}[m, n]$. On the other hand, high-magnitude values of $\hat{x}_{1}[m, n]$, possibly due the presence of structures, should be enhanced. A pointwise nonlinearity performing the said operations is given in the following:

$$
\begin{gathered}
\tilde{x}_{1}[m, n]=\left(1+\frac{1}{\alpha}\right) \cdot \hat{x}_{1}[m, n] \cdot g\left(\left|\hat{x}_{1}[m, n]\right|\right), \\
g(\cdot)=1+\gamma \cdot \sqrt{1+\alpha} \cdot \exp \left[-\frac{(\cdot)^{2}}{2} \cdot \frac{\alpha}{(1+1 / \alpha)}\right] .
\end{gathered}
$$

The magnitude of (16) is plotted in Figure 11 for different values of the parameter $\alpha$.

The low-gain zone near the origin is controlled by the parameter $\gamma$; the parameter $\alpha$ controls the enhancement effect on the edges. Both parameters are set empirically. The nonlinearity (16) has been presented in [14], where the analogy of this nonlinearity with the Bayesian estimator of spiky images in Gaussian observation noise is discussed.

To sum up, the adopted nonlinearity is locally tuned to the image characteristics. When the presence of an edge is detected, an edge thinning in the local RT of the edge image is performed. This operation, which encompasses a spatial 


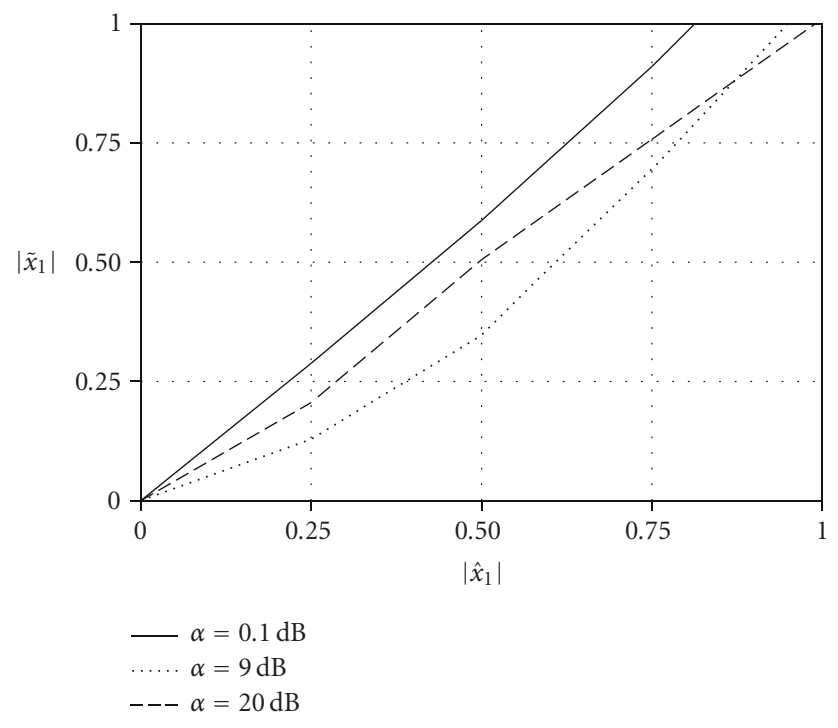

FIGURE 11: Nonlinearity given by (16), employed for natural images deblurring and parameterized with respect to the parameter $\alpha$ for $\gamma=0.5$.
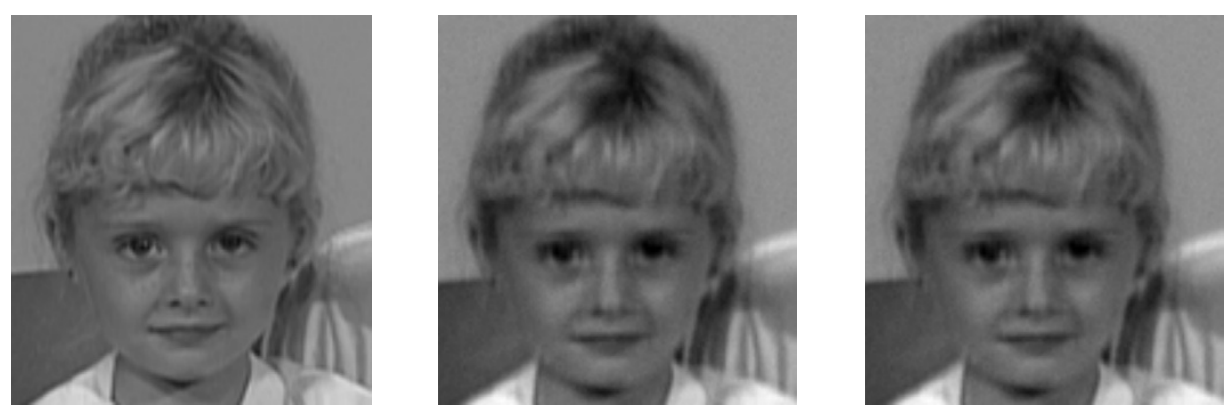

FIGURE 12: Daughter image. From left to right: original image; linear estimation $\hat{x}^{(1)}[m, n]$; nonlinear estimation $\tilde{x}^{(1)}[m, n]$ after the first iteration.

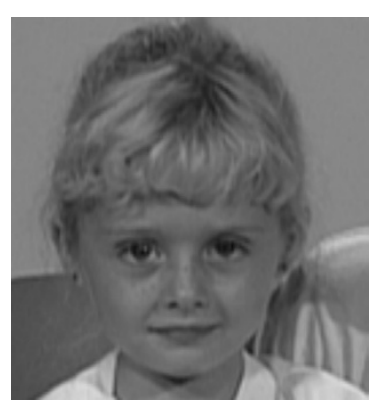

FIGURE 13: Daughter image.

memory in the edge enhancement, is performed directly in the RT domain since the image edges are compactly represented in this domain. When an edge structure is not detected, which may happen for example in textured or uniform regions, the adopted nonlinearity reduces to a pointwise edge enhancement. It is worth pointing out that, as diffusely discussed in [16], the compact representation of an edge in the RT domain is related to the tuning between the size of the local RT transform and the bandpass of the edge extracting filter.

After the nonlinear estimate $\tilde{x}_{1}[m, n]$ has been computed, the estimate $\tilde{x}[m, n]$ is obtained by reconstructing through the inverse filter bank $\phi^{(0)}[m, n]$ and $\phi^{(1)}[m, n]$, that is, (see Figure 3)

$$
\tilde{x}[m, n]=\left(\phi^{(0)} * \hat{x}_{0}\right)[m, n]+\left(\phi^{(1)} * \tilde{x}_{1}\right)[m, n] .
$$

We remark that the nonlinear estimator modifies only the edge image magnitude, leaving the phase restoration to the linear estimation stage, performed by means of the filter bank $f_{i}^{(k+1)}[m, n], i=0, \ldots, M-1$. The action of the nonlinearity on a natural image is presented in Figure 12, where along with the original image, the linear estimation $\hat{x}^{(1)}[m, n]$, and the nonlinear estimation $\tilde{x}^{(1)}[m, n]$ obtained after the first iteration are shown.

\section{EXPERIMENTAL RESULTS}

In Figures 9, 10, and 13, some of the images we have used for our experimentations are reported. The images are blurred 

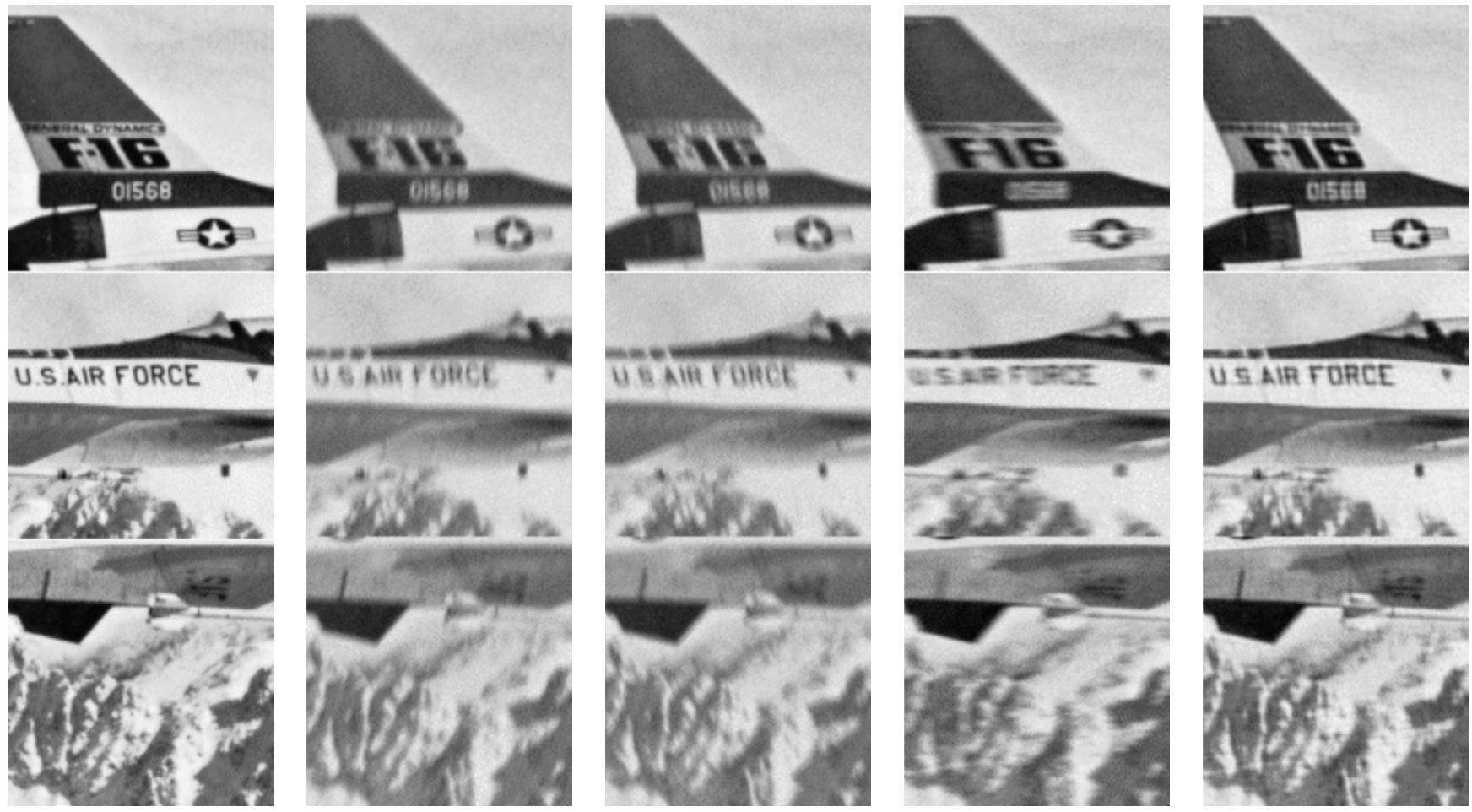

FIgURE 14: F16 image. First column: details of the original image. Second, third, and fourth columns: blurred observations of the original details. Fifth column: restored details $(\mathrm{SNR}=20 \mathrm{~dB})$.
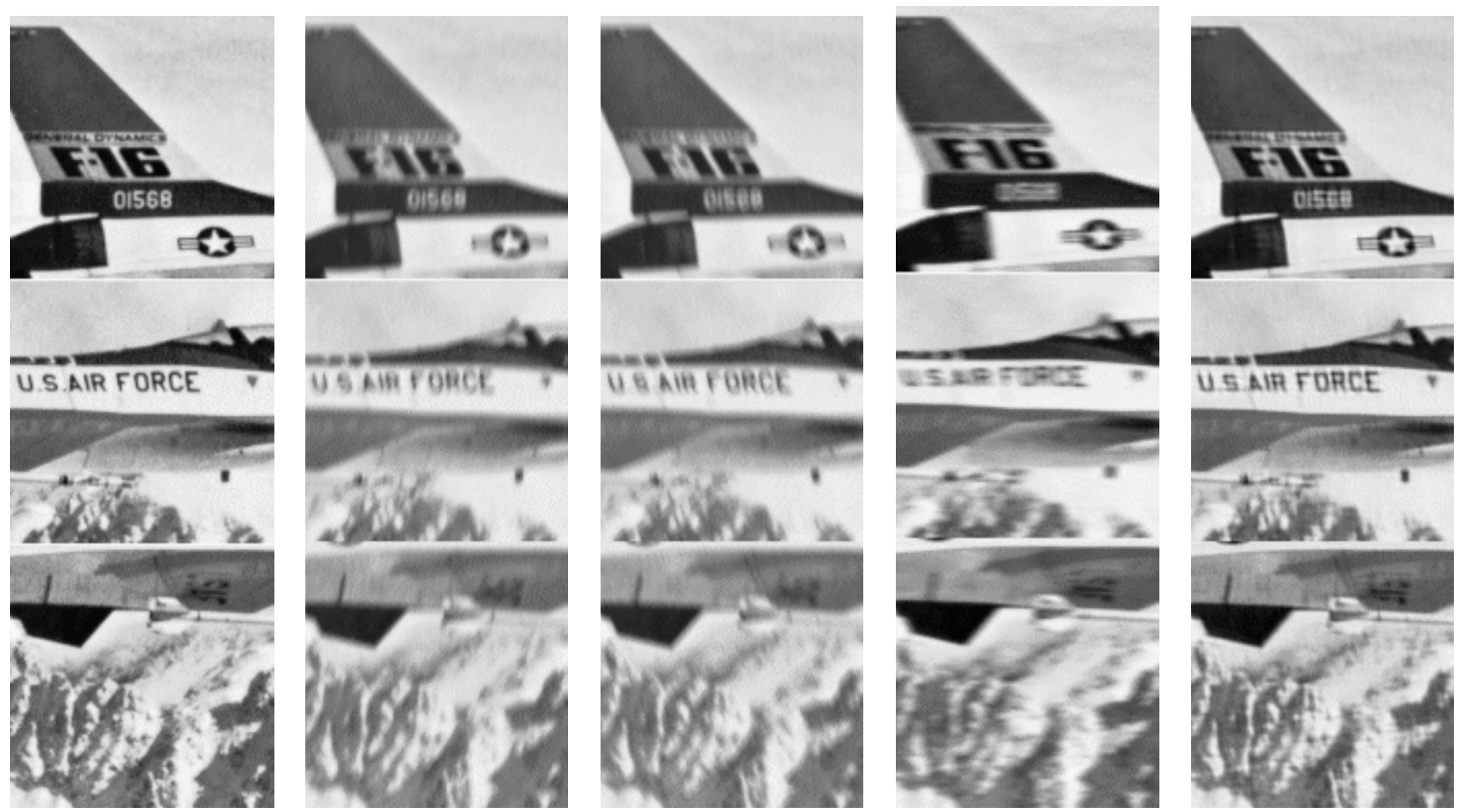

FIGURE 15: F16 image. First column: details of the original image. Second, third, and fourth columns: blurred observations of the original details. Fifth column: restored details $(\mathrm{SNR}=40 \mathrm{~dB})$. 


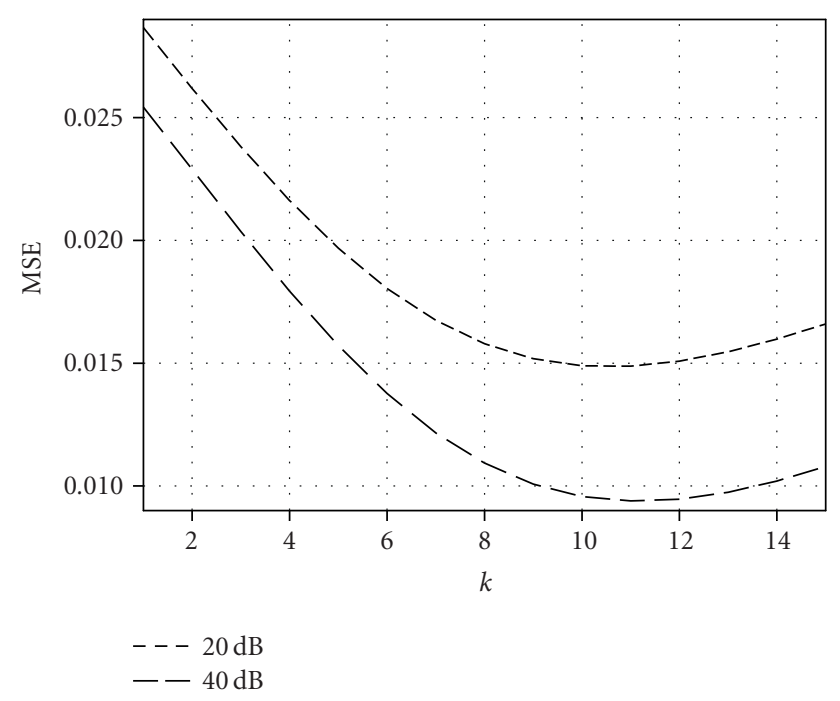

FIGURE 16: F16 image: mean square error versus the iterations number.

using the blurring filters having the following impulse responses:

$$
\begin{gathered}
h_{1}[m, n]=\left[\begin{array}{lllllll}
0 & 0 & 0 & 1 & 0 & 0 & 0 \\
0 & 0 & 0 & 1 & 0 & 0 & 0 \\
0 & 0 & 0 & 1 & 0 & 0 & 0 \\
0 & 0 & 0 & 1 & 0 & 0 & 0 \\
0 & 0 & 1 & 0 & 0 & 0 & 0
\end{array}\right], \\
h_{2}[m, n]=\left[\begin{array}{lllllll}
0 & 0 & 0 & 1 & 0 & 0 & 0 \\
0 & 0 & 0 & 1 & 0 & 0 & 0 \\
0 & 0 & 0 & 1 & 0 & 0 & 0 \\
0 & 0 & 1 & 0 & 0 & 0 & 0 \\
0 & 0 & 1 & 0 & 0 & 0 & 0
\end{array}\right], \\
h_{3}[m, n]=\left[\begin{array}{cccccccc}
0 & 0 & 0 & 0 & 0 & 0 & 0 \\
0 & 0 & 0 & 0 & 0 & 0 & 0 \\
0.5 & 0.86 & 0.95 & 1 & 0.95 & 0.86 & 0.5 \\
0 & 0 & 0 & 0 & 0 & 0 & 0 \\
0 & 0 & 0 & 0 & 0 & 0 & 0
\end{array}\right] .
\end{gathered}
$$

In Figure 14, some details belonging to the original image shown in Figure 9 are depicted. The corresponding blurred observations, affected by additive white Gaussian noise at SNR $=20 \mathrm{~dB}$, obtained using the aforementioned blurring filters, are also shown along with the deblurred images. In Figure 15, the same images are reported for blurred images affected by additive white Gaussian noise at $\mathrm{SNR}=40 \mathrm{~dB}$. In Figure 16, the MSE, defined as

$$
\operatorname{MSE} \stackrel{\text { def }}{=} \frac{1}{N^{2}} \sum_{i, j=0}^{N-1}(x[i, j]-\hat{x}[i, j])^{2},
$$

is plotted versus the iterations number at different SNR values for the deblurred image.
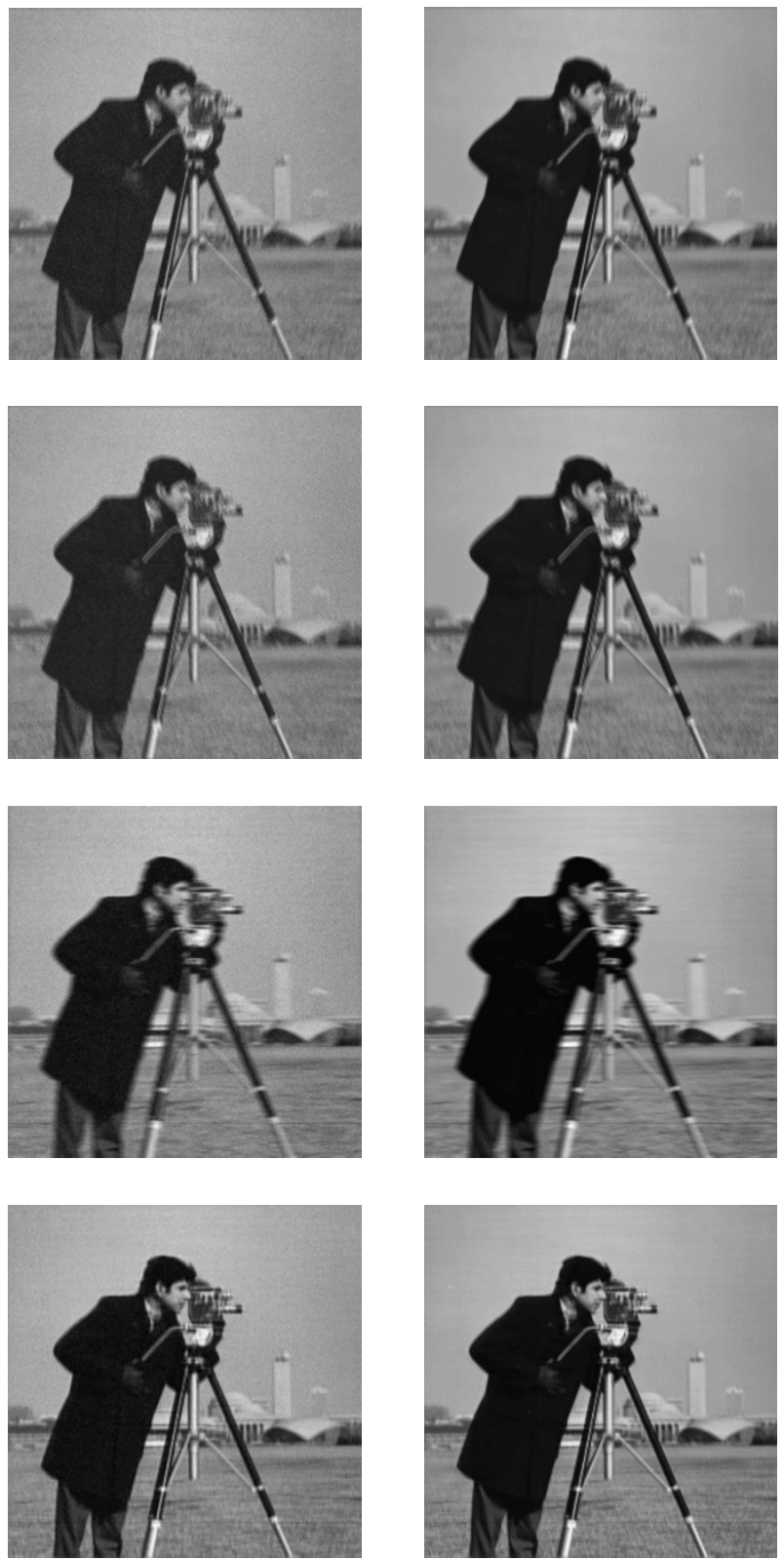

Figure 17: Cameraman image. Left column, first, second, and third row: observations. Fourth row: restored image $($ SNR $=20 \mathrm{~dB})$. Right column, first, second, and third rows: observations. Fourth row: restored image $(\mathrm{SNR}=40 \mathrm{~dB})$.

Similar results are reported in Figure 17 for the image shown in Figure 10 and the corresponding MSE is shown in Figure 18.

Moreover, in Figure 19, along with the restored version of the image depicted in Figure 13 obtained using the proposed method, the corresponding restored images obtained using the method introduced by the authors in [14] are reported. Eventually, in Figure 20, the MSE versus the number of iterations is plotted for both the proposed method and the one presented in [14]. 


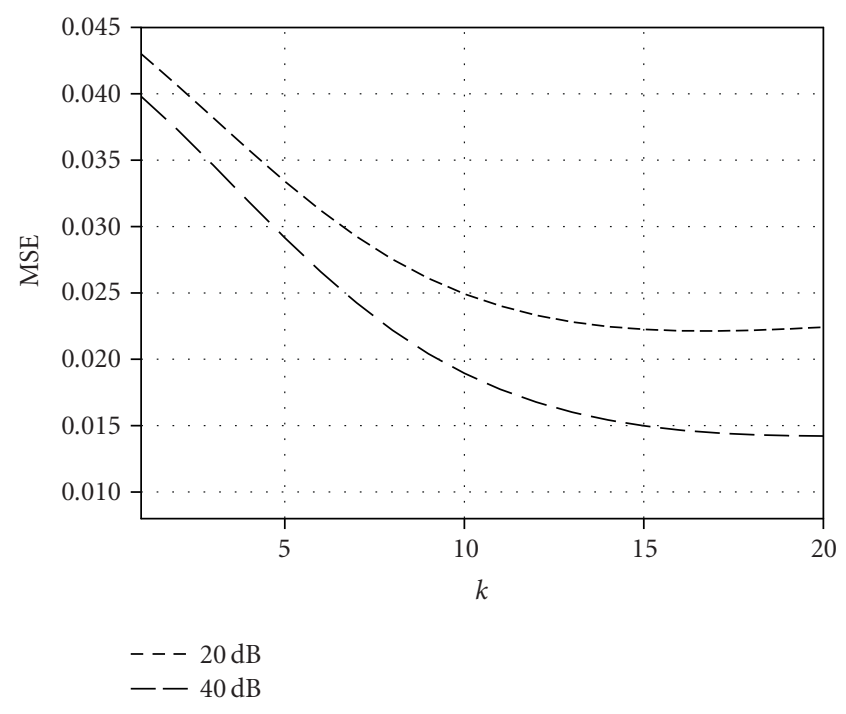

FIGURE 18: Cameraman image: mean square error versus the iterations number.

Note that the algorithm converges in few iterations toward a local minimum and then may even diverge. In fact, as well known [17], the global convergence of the Bussgang algorithm cannot be guaranteed. The choice of the desired restoration result can be made by visual inspection of the restored images obtained during the iterations, which is, as also pointed out in [6], typical of blind restoration techniques.

It is worth noting that the deblurring algorithm gives images of improved visual quality, thus significantly reducing the distance, in the mean square sense, from the original unblurred image.

\section{CONCLUSION}

This work describes an algorithm for blind image restoration that iteratively performs a linear and a nonlinear processing. The nonlinear stage is based on a novel nonlinear processing technique that is based on a compact representation of the image edges by means of a local Radon transform. In order to focus the blurred image edges, and to drive the overall blind restoration algorithm to a sharp, focused image, a suitable nonlinear processing is designed in the Radon transformed domain. The nonlinear processing is spatially variant, depending on the detection of strong edges versus uniform or textured regions.

The performance of the algorithm is assessed by a number of experiments showing the overall quality of the restored images.

\section{APPENDIX}

\section{MULTICHANNEL BUSSGANG ALGORITHM}

The multichannel Bussgang blind deconvolution algorithm outlined in [14] is here briefly summarized.
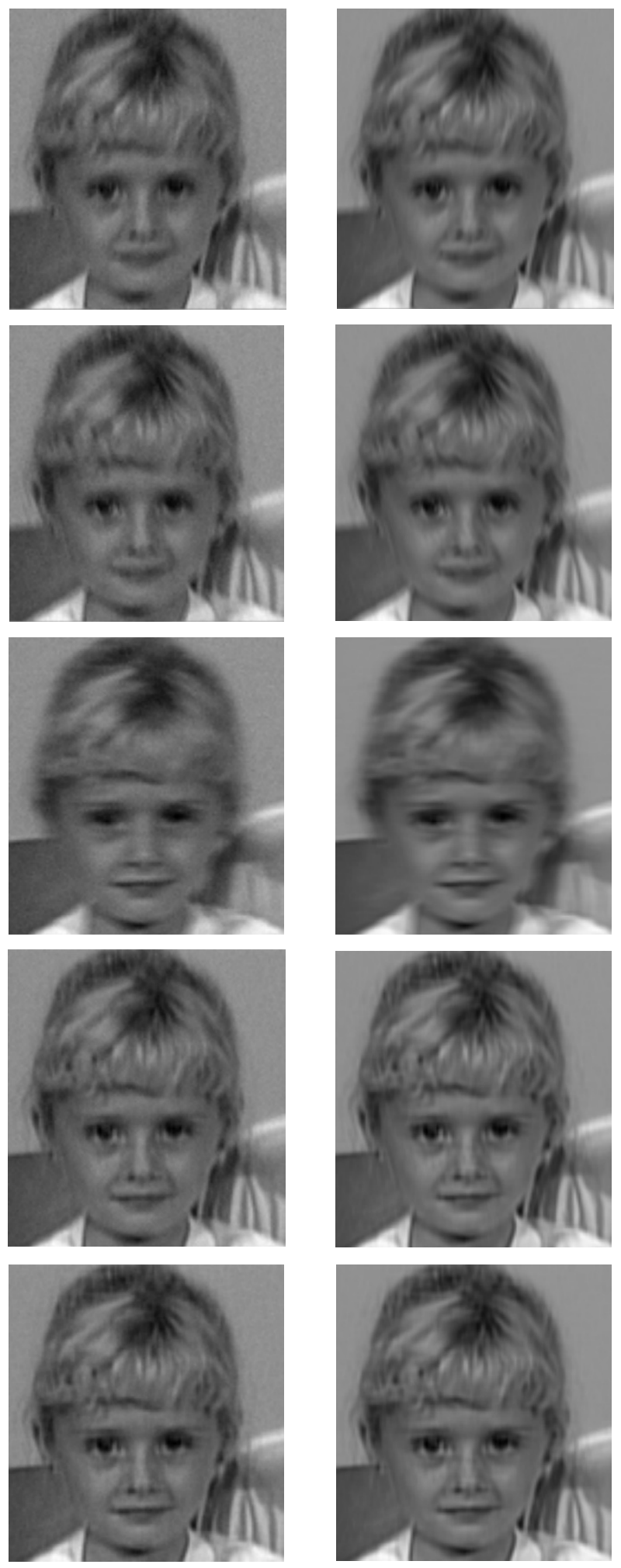

FIGURE 19: Daughter image. Left column, first, second, and third row: observations. Fourth row: restored image using the method proposed in [14] $(\mathrm{SNR}=20 \mathrm{~dB})$. Fifth row: restored image using the proposed method (SNR $=20 \mathrm{~dB}$ ). Right column, first, second, and third rows: observations. Fourth row: restored image using the method proposed in [14] $(\mathrm{SNR}=40 \mathrm{~dB})$. Fifth row: restored image using the proposed method $(\mathrm{SNR}=40 \mathrm{~dB})$. 


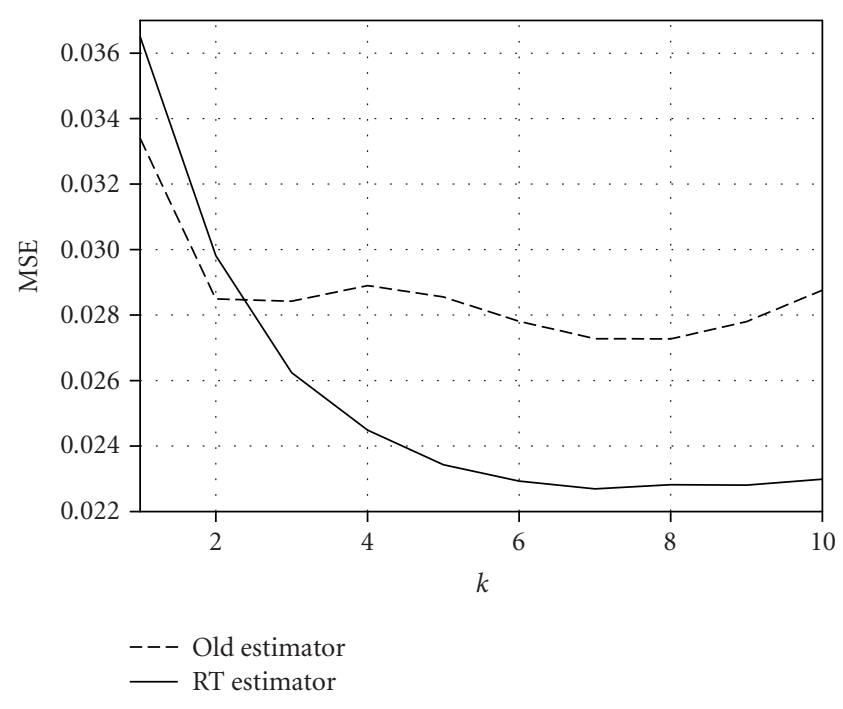

FIGURE 20: Daughter image: comparison between the mean square error of the old estimator [14] and of the proposed estimator ("RT Estimator") versus the iterations number.

With reference to Figure 2, the $k$ th iteration of the blind deconvolution algorithm is constituted by the following steps.

(1) Linear estimation step. The deconvolved image $\hat{x}^{(k)}[m, n]$ is computed from the observations set $y_{0}, \ldots, y_{M-1}$ by filtering through a previous estimate of the Wiener filter bank $f_{i}^{(k-1)}[m, n], i=0, \ldots, M-1$, that is,

$$
\hat{x}^{(k)}[m, n]=\sum_{i=0}^{M-1}\left(f_{i}^{(k-1)} * y_{i}\right)[m, n] .
$$

Since the original image can be retrieved except for an amplitude scale factor, at each iteration, the Wiener filter bank is normalized to yield a unitary energy output.

(2) Nonlinear estimation step. According to a suitable stochastic model of the image $x[m, n]$, the nonlinear MMSE estimate $\tilde{x}^{(k)}[m, n]$ is computed through the conditional a posteriori expectation:

$$
\tilde{x}^{(k)}[m, n]=\eta\left(\hat{x}^{(k)}\right) \stackrel{\text { def }}{=} \mathrm{E}\left\{x[m, n] \mid \hat{x}^{(k)}\right\}
$$

In general, the nonlinear estimator $\eta(\cdot)$ exhibits nonzero spatial memory.

(3) Wiener filter coefficients update step. The Wiener filter bank $f_{i}^{(k)}[m, n], i=0, \ldots, M-1$, is recomputed by solving the $M \times(2 P+1)^{2}$ normal equations for the determination of the $M \times(2 P+1)^{2}$ unknowns:

$$
\begin{aligned}
& \sum_{j=0}^{M-1} \sum_{t, u=-P}^{P} R_{y_{j} y_{i}}[r-t, s-u] f_{j}^{(k)}[t, u] \\
& =R_{\tilde{x}^{(k-1)} y_{i}}[r, s] \text { for }\left\{\begin{array}{l}
i=0, \ldots, M-1, \\
r, s=-P, \ldots, P,
\end{array}\right.
\end{aligned}
$$

where the cross-correlations functions $R_{y_{j}, y_{i}}[r, s] \stackrel{\text { def }}{=}$ $\mathrm{E}\left\{y_{j}[m, n] y_{i}[m-r, n-s]\right\}$ and $R_{\tilde{x}^{(k-1)}, y_{i}}[r, s] \stackrel{\text { def }}{=}$ $\mathrm{E}\left\{\tilde{x}^{(k-1)}[m, n] y_{i}[m-r, n-s]\right\}$ are substituted by their sample estimates $\hat{R}_{y_{j} y_{i}}[r, s]$ and $\hat{R}_{\tilde{x}^{(k-1)} y_{i}}[r, s]$, respectively. We must outline that in this step, only the crosscorrelations $\hat{R}_{\tilde{x}^{(k-1)} y_{i}}[r, s]$ are computed since $\hat{R}_{y_{j} y_{i}}[r, s]$ are computed only once before starting the iterations.

(4) Convergence test step. Convergence is tested by a suitable criterion.

The algorithm is initialized by a suitable initial guess $f_{i}^{(0)}[m, n], i=0, \ldots, M-1$, of the Wiener filter bank.

As outlined in $[17,18]$, the iterative algorithm reaches an equilibrium point, namely, $f_{i}^{(k-1)}[m, n]=f_{i}^{(k)}[m, n]$, when the cross-correlations between the deconvolved image $\hat{x}^{(k)}[m, n]$ and the measured images $y_{i}[m, n], i=0, \ldots, M-1$, become proportional to the cross-correlations between the estimate $\tilde{x}^{(k)}[m, n]$ and the observations, that is,

$$
R_{\hat{x}^{(k)} y_{i}}[r, s]=\text { const } \cdot R_{\tilde{x}^{(k)} y_{i}}[r, s] .
$$

Some of the authors have presented a discussion on the convergence of the algorithm in [26], where the analysis is conducted in an error energy reduction sense for the singlechannel case. The condition for convergence given in [26] straightforwardly extends to the multichannel case since the variables involved in the analysis (original image, deconvolved image, and nonlinear estimate) do not change.

\section{REFERENCES}

[1] R. Molina, "On the hierarchical Bayesian approach to image restoration: applications to astronomical images," IEEE Trans. on Pattern Analysis and Machine Intelligence, vol. 16, no. 11, pp. 1122-1128, 1994.

[2] J. P. Muller, Ed., Digital Image Processing in Remote Sensing, Taylor \& Francis, Philadelphia, Pa, USA, 1988.

[3] T. Wilson and S. J. Hewlett, "Imaging strategies in threedimensional confocal microscopy," in Proc. SPIE Biomedical Image Processing, A. C. Bovik and W. E. Higgins, Eds., vol. 1245 of Proceedings of SPIE, pp. 35-45, San Jose, Calif, USA, February 1991.

[4] N. P. Galatsanos, V. Z. Mesarović, R. Molina, and A. K. Katsaggelos, "Hierarchical Bayesian image restoration from partially known blurs," IEEE Trans. Image Processing, vol. 9, no. 10, pp. 1784-1797, 2000.

[5] A. K. Katsaggelos, Ed., Digital Image Restoration, SpringerVerlag, New York, NY, USA, 1991.

[6] D. Kundur and D. Hatzinakos, "Blind image deconvolution," IEEE Signal Processing Magazine, vol. 13, no. 3, pp. 43-64, 1996. 
[7] G. Harikumar and Y. Bresler, "Perfect blind restoration of images blurred by multiple filters: theory and efficient algorithms," IEEE Trans. Image Processing, vol. 8, no. 2, pp. 202 219, 1999.

[8] G. B. Giannakis and R. W. Heath Jr., "Blind identification of multichannel FIR blurs and perfect image restoration," IEEE Trans. Image Processing, vol. 9, no. 11, pp. 1877-1896, 2000.

[9] M. Belge, M. E. Kilmer, and E. L. Miller, "Wavelet domain image restoration with adaptive edge-preserving regularization," IEEE Trans. Image Processing, vol. 9, no. 4, pp. 597-608, 2000.

[10] M. C. Robini and I. E. Magnin, "Stochastic nonlinear image restoration using the wavelet transform," IEEE Trans. Image Processing, vol. 12, no. 8, pp. 890-905, 2003.

[11] D. P. K. Lun, T. C. Hsung, and T. W. Shen, "Orthogonal discrete periodic Radon transform. Part II: applications," Signal Processing, vol. 83, no. 5, pp. 957-971, 2003.

[12] R. G. Lane and R. H. T. Bates, "Relevance for blind deconvolution of recovering Fourier magnitude from phase," Optics Communications, vol. 63, no. 1, pp. 11-14, 1987.

[13] P. Campisi, S. Colonnese, G. Panci, and G. Scarano, "Multichannel Bussgang algorithm for blind restoration of natural images," in Proc. IEEE International Conference on Image Processing (ICIP '03), vol. 2, pp. 985-988, Barcelona, Spain, September 2003.

[14] G. Panci, P. Campisi, S. Colonnese, and G. Scarano, "Multichannel blind image deconvolution using the Bussgang algorithm: spatial and multiresolution approaches," IEEE Trans. Image Processing, vol. 12, no. 11, pp. 1324-1337, 2003.

[15] S. R. Deans, The Radon Transform and Some of Its Applications, Krieger Publishing, Malabar, Fla, USA, 1993.

[16] J.-L. Starck, E. J. Candes, and D. L. Donoho, "The curvelet transform for image denoising," IEEE Trans. Image Processing, vol. 11, no. 6, pp. 670-684, 2002.

[17] R. Godfrey and F. Rocca, "Zero memory nonlinear deconvolution," Geophysical Prospecting, vol. 29, pp. 189-228, 1981.

[18] S. Bellini, "Bussgang techniques for blind deconvolution and equalization," in Blind Deconvolution, S. Haykin, Ed., pp. 8 59, Prentice-Hall, Englewood Cliffs, NJ, USA, 1994.

[19] G. Jacovitti, G. Panci, and G. Scarano, "Bussgang-zero crossing equalization: an integrated HOS-SOS approach," IEEE Trans. Signal Processing, vol. 49, no. 11, pp. 2798-2812, 2001.

[20] A. Neri, G. Scarano, and G. Jacovitti, "Bayesian iterative method for blind deconvolution," in Proc. SPIE Adaptive Signal Processing, vol. 1565 of Proceedings of SPIE, pp. 196-208, San Diego, Calif, USA, July 1991.

[21] G. Jacovitti and A. Neri, "Multiresolution circular harmonic decomposition," IEEE Trans. Signal Processing, vol. 48, no. 11, pp. 3242-3247, 2000.

[22] G. Jacovitti and A. Neri, "Multiscale image features analysis with circular harmonic wavelets," in Proc. SPIE Wavelet Applications in Signal and Image Processing III, vol. 2569 of Proceedings of SPIE, pp. 363-374, San Diego, Calif, USA, July 1995.

[23] C. A. Berenstein and E. V. Patrick, "Exact deconvolution for multiple convolution operators-an overview, plus performance characterizations for imaging sensors," Proceedings of the IEEE, vol. 78, no. 4, pp. 723-734, 1990.

[24] J. L. Starck, "Image processing by the curvelet transform," in Proc. Tyrrhenian International Workshop on Digital Communications (IWDC '02), Capri, Italy, September 2002.

[25] D. L. Donoho and M. R. Duncan, "Digital curvelet transform: strategy, implementation, and experiments," in Proc. SPIE Wavelet Applications VII, vol. 4056 of Proceedings of SPIE, pp. 12-30, Orlando, Fla, USA, April 2000.

[26] P. Campisi and G. Scarano, "A multiresolution approach for texture synthesis using the circular harmonic functions," IEEE Trans. Image Processing, vol. 11, no. 1, pp. 37-51, 2002.
Stefania Colonnese was born in Rome, Italy. She received the "Laurea" degree in electronic engineering from the Università degli Studi di Roma “La Sapienza," Italy, in 1993, and the Ph.D. degree in electronic engineering from the Università degli Studi di Roma "Roma Tre" in 1997. In 1993, she joined the Fondazione Ugo Bordoni, Rome, first as a scholarship holder, and later as an Associate Researcher. During the MPEG-

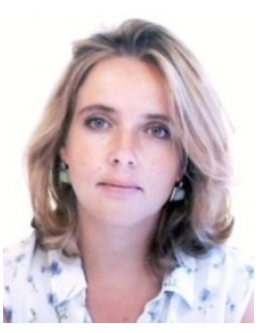
4 standardization activity, she was involved in the MPEG-4 N2 core experiment on automatic video segmentation. In 2001, she joined the Dipartimento di Scienza e Tecnica dell'Informazione e della Comunicazione (Infocom), Università degli Studi di Roma "La Sapienza," as an Assistant Professor. Her research interests lie in the areas of video communications, and image and signal processing.

Patrizio Campisi received the "Laurea" degree in electronic engineering (summa cum laude) from the Università degli Studi di Roma "La Sapienza," Italy, and the Ph.D. degree in electronic engineering from the Università degli Studi di Roma "Roma Tre," Italy, in 1995 and 1999, respectively. He is an Assistant Professor in the Dipartimento di Elettronica Applicata, Università degli Studi di Roma "Roma Tre," where he has also been a lecturer for the graduate course "Signal Theory" since 1998. From September 1997 until April 1998, he was a Visiting Research Associate with the Communication Laboratory, University of Toronto, Toronto, Canada, and from July 2000 until December 2000, he was a Postdoctoral Fellow with the same laboratory. From October 1999 to October 2001, he held a Postdoctoral position at the Università degli Studi di Roma “Roma Tre.” From March 2003 until June 2003, he was a Visiting Researcher at the Beckman Institute, University of Illinois at Urbana-Champaign, Ill, USA. His research interests lie in the area of digital signal and image processing with applications to multimedia communications. Dr. Campisi has been a member of the Technical Committees of several IEEE conferences. He was the organizer of the special session on "Texture analysis and synthesis" for the IEEE International Symposium on Image and Signal Processing and Analysis 2003 (ISPA 2003).

Gianpiero Panci was born in Palestrina, Rome, Italy. He received the "Laurea" degree in telecommunications engineering in 1996 and the Ph.D. in communication and information theory in 2000, both from Università "La Sapienza," Italy. He worked in the area of signal processing for communication and radar systems, and in 1999, he was involved in the design of the signum coded SAR proces-

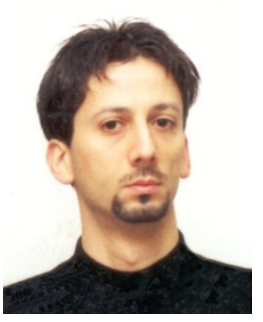
sor for the shuttle radar topographic mission. He currently holds an Associate Research position at the Dipartimento di Scienza e Tecnica dell'Informazione e della Comunicazione (Infocom), Università degli Studi di Roma "La Sapienza," where he is also a Lecturer for a graduate course in communications. His current research interests include statistical signal processing, blind identification and equalization, and array processing. 
Gaetano Scarano was born in Campobasso, Italy. He received the "Laurea" degree in electronic engineering from Università degli Studi di Roma "La Sapienza," Italy, in 1982. In 1982, he joined the Istituto di Acustica, Consiglio Nazionale delle Ricerche, Rome, Italy, as Associate Researcher. Since 1988, he has been teaching digital signal processing at Università degli Studi di Perugia, Perugia, Italy, where in 1991 he became Asso-

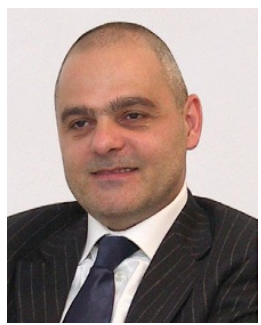
ciate Professor of signal theory. In 1992, he joined the Dipartimento di Scienza e Tecnica dell'Informazione e della Comunicazione (Infocom), Università degli Studi di Roma "La Sapienza," first as an Associate Professor of image processing, then as a Professor of signal theory. His research interests lie in the areas of communications, signal and image processing, and estimation and detection theory, and include channel equalization and estimation, texture synthesis, and image restoration. He has served as an Associate Editor of the IEEE Signal Processing Letters. 